\title{
Nonthermal ablation in the rat brain using focused ultrasound and an ultrasound contrast agent: long-term effects
}

\author{
Nathan McDannold, PhD, Yongzhi Zhang, MD, and Natalia Vykhodtseva, PhD \\ Department of Radiology, Brigham and Women's Hospital, Harvard Medical School, Boston, Massachusetts
}

OBJECTIVE Thermal ablation with transcranial MRI-guided focused ultrasound (FUS) is currently under investigation as a less invasive alternative to radiosurgery and resection. A major limitation of the method is that its use is currently restricted to centrally located brain targets. The combination of FUS and a microbubble-based ultrasound contrast agent greatly reduces the ultrasound exposure level needed to ablate brain tissue and could be an effective means to increase the "treatment envelope" for FUS in the brain. This method, however, ablates tissue through a different mechanism: destruction of the microvasculature. It is not known whether nonthermal FUS ablation in substantial volumes of tissue can safely be performed without unexpected effects. The authors investigated this question by ablating volumes in the brains of normal rats.

METHODS Overlapping sonications were performed in rats $(n=15)$ to ablate a volume in 1 hemisphere per animal. The sonications (10-msec bursts at $1 \mathrm{~Hz}$ for 60 seconds; peak negative pressure $0.8 \mathrm{MPa}$ ) were combined with the ultrasound contrast agent Optison $(100 \mu \mathrm{l} / \mathrm{kg})$. The rats were followed with MRI for 4-9 weeks after FUS, and the brains were examined with histological methods.

RESULTS Two weeks after sonication and later, the lesions appeared as cyst-like areas in T2-weighted MR images that were stable over time. Histological examination demonstrated well-defined lesions consisting of a cyst-like cavity that remained lined by astrocytic tissue. Some white matter structures within the sonicated area were partially intact.

CONCLUSIONS The results of this study indicate that nonthermal FUS ablation can be used to safely ablate tissue volumes in the brain without unexpected delayed effects. The findings are encouraging for the use of this ablation method in the brain.

http://thejns.org/doi/abs/10.3171/2015.10.JNS151525

KEY WORDS focused ultrasound; ablation; brain

$\mathrm{T}$ HERMAL ablation via MRI-guided focused ultrasound (FUS) is an emerging minimally invasive alternative to resection and radiosurgery. ${ }^{4,18,23}$ While the use of FUS ablation has a long history, $3,26,32$ the advent of large-scale phased array systems and aberration correction methods that have enabled transmission through the intact human skull $2,7,8$ and MRI methods to quantify temperature change ${ }^{21}$ have renewed interest in this technology for use in the brain. Transcranial MRI-guided FUS is now being investigated clinically for thermal ablation of tumors 9,29 and for functional neurosurgery applications..$^{10,22,27,28}$
The most serious current limitation on this technology is that ablation targets cannot be placed near the skull bone, restricting the "treatment envelope" to deep, centrally located targets in the brain. The margin between achieving a sufficient focal temperature rise and maintaining a safe temperature in the bone, which is highly absorbing of ultrasound, is not large. High-energy densities in the bone occur when the focal region is placed away from central targets, due to limited transmission through the bone when the angle of incidence of the acoustic field at the bone interface is large ${ }^{44}$ Furthermore, intraindividual variations

ABBREVIATIONS ETL = echo train length; FOV = field of view; FSE = fast spin echo; FUS = focused ultrasound; $\mathrm{H}$ \& $\mathrm{E}=$ hematoxylin and eosin; $\mathrm{LFB}=\mathrm{Luxol}$ fast blue. SUBMITTED June 30, 2015. ACCEPTED October 23, 2015. 
in skull properties are significant, and high focal temperature changes cannot be achieved in all individuals, even at ideal targets such as the thalamus. ${ }^{24}$ Having a way to increase the effects at the focus while reducing the overall energy delivery could expand the use of FUS in the brain and increase the number of patients who can benefit from this less-invasive surgical technique.

An effective means to enhance the energy delivery at the focal point is to combine the FUS exposures (sonications) with an intravenous injection of a microbubble-based ultrasound imaging contrast agent. ${ }^{6,13,14,33,38,40,41,45}$ Since gas is compressible (and water is nearly incompressible), these microbubbles in circulation can respond strongly to an acoustic pressure wave. Their oscillation and collapse within the ultrasound field produces a number of mechanical effects that are concentrated on the vasculature and can be exploited for therapeutic use. These effects can be violent enough to damage and destroy capillaries, leading to infarct and tissue necrosis. Since the pressure amplitude necessary for this nonthermal ablation approach is very low, an order of magnitude below what is required for thermal ablation, it has been of particular interest for increasing the use of FUS in the brain ${ }^{19,30,31,42}$ and for the treatment of brain tumors. ${ }^{5}$

Unlike thermal ablation, where high temperatures immediately coagulate tissue, necrosis is delayed with this type of ablation. ${ }^{42}$ Furthermore, the necrotic tissue is removed rapidly, whereas "cooked" tissue is not as easily accessed by macrophages and its removal can be a slow process. The rapid breakdown and removal of tissue after nonthermal ablation with FUS and microbubbles may pose unknown risks, particularly when large tissue volumes are ablated. Damaged blood vessels at the margin of the ablated region might be at risk for failing at a later time, leading to further downstream effects. As the ischemic tissue dies and is removed via macrophages, by-products may be released that could lead to toxicity or progressive necrosis in surrounding tissues.

The progression of this type of lesion might be expected to follow a similar course as ischemic stroke. ${ }^{11,17}$ In areas where blood flow falls below a critical value $(\sim 20 \%$ of pre-ischemia values), tissue death occurs within a few minutes after the onset of ischemia. During a subacute phase, irreversible damage expands into areas that had reduced perfusion (between $25 \%-50 \%$ of pre-ischemia values) over several hours. Finally, a delayed phase of injury can occur over the following days or weeks where secondary effects such as vasogenic edema, apoptosis, and inflammation can occur, leading to further progression of tissue death. Radiation-induced necrosis can also lead to a vicious cycle where a lesion can progressively enlarge over time with mass effect, and it is thought that this late-stage necrosis arises from earlier vascular necrosis. ${ }^{25}$ While earlier studies on FUS ablation enhanced by circulating microbubbles have examined both short- and long-term effects, ${ }^{30}$ it remains to be seen whether substantial tissue volumes can safely be ablated without delayed effects or progressive tissue necrosis. It is also unknown whether contiguous volumes can be ablated by sonicating at overlapping targets, or if residual bubble fragments influence the outcome of subsequent exposures. ${ }^{12}$ Such "cavitation memory" has been previously observed in studies that did not use preformed microbubble agents. ${ }^{43}$ It is also possible that vascular damage at one target reduces blood flow and thus microbubble concentration, at downstream adjacent regions.

The purpose of this study was therefore to examine long-term effects in the brain after nonthermal ablation with FUS and circulating microbubbles ablation. Brain tissue volumes in normal rats were ablated by sonicating 4 overlapping targets in combination with the microbubble ultrasound imaging contrast agent Optison. The tissue effects were then monitored for 1 month or more using MRI, and the brains were examined with histological methods.

\section{Methods \\ Animals}

All experiments were done in accordance with procedures approved by the Harvard Medical School Institutional Animal Care and Use Committee. The animals were housed, fed, and watered according to the Office of Laboratory Animal Welfare and the Association for Assessment and Accreditation of Laboratory Care regulations. Lesions were created transcranially in 15 male Sprague-Dawley rats (262-433g). Before each procedure, the animals were anesthetized with an intraperitoneal injection of ketamine $(90 \mu \mathrm{g} / \mathrm{kg})$ and xylazine $(10 \mu \mathrm{g} / \mathrm{kg})$ administered hourly or as needed. The fur on the head was removed using clippers and depilatory cream, and a catheter was placed in the tail vein.

\section{MRI-Guided FUS}

FUS exposures (10-msec bursts applied at $1 \mathrm{~Hz}$ for 300 seconds) were delivered immediately after the administration of the microbubble ultrasound contrast agent Optison (GE Healthcare) to induce tissue necrosis under MRI guidance. The transcranial sonications were applied using a 1.1-MHz FUS transducer driven with a function generator (33220A, Agilent) and amplifier (240 L, E\&I). Electrical power output was measured using a power meter (E4419B, Agilent) and dual-directional coupler (C5948-10, Werlatone). The transducer was mounted on a manually operated, 3-axis MRI-compatible positioning system (Fig. 1). Acoustic coupling between the FUS transducer and the rat's head was achieved with deionized and degassed water. A $4 \times 5.5-\mathrm{cm}$-diameter transmit/receive surface coil was placed under the rat's head, and the system was placed in a 4.7-T animal MRI (Bruker). We used a spherically

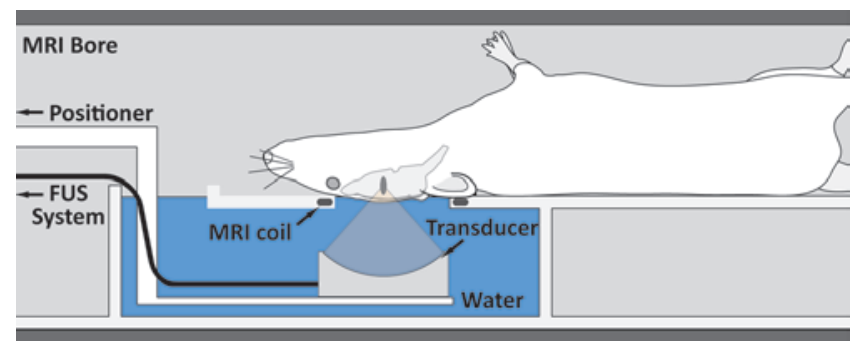

FIG. 1. Experimental setup. The experiments were performed with a FUS insert developed for a 4.7-T animal MRI. Figure is available in color online only. 
curved transducer with a diameter and radius of curvature of 4 and $3 \mathrm{~cm}$, respectively. The transducer was calibrated using a radiation force balance to measure the acoustic power, and scans of the acoustic intensity were obtained with a $0.2-\mathrm{mm}$-diameter needle hydrophone (HNC-0200, Onda). These calibrations were used to estimate the peak negative pressure amplitude at the focus in water. ${ }^{20}$ The acoustic power was $0.86 \mathrm{~W}$, which produced a pressure amplitude in water of $1.3 \mathrm{MPa}$. Assuming approximately $65 \%$ insertion loss at this frequency through the rat skull ${ }^{36}$ and an attenuation coefficient in brain of $5 \mathrm{~Np} / \mathrm{m} / \mathrm{MHz},{ }^{15}$ the negative peak pressure amplitude in the brain was estimated to be $0.8 \mathrm{MPa}$. These exposure levels were determined in a pilot study in the first animal where sonications at increasing power levels were performed until a hypointense spot was observed in T2*-weighted MRI. The width and length of the $50 \%$ isopressure contours of the 2 transducers were 1.5 and $7.4 \mathrm{~mm}$, respectively. The length of the focal region was sufficient to ablate the entire thickness of the rat brain from the skull base to the cortex. The transducer, MRI coil, and positioning system were each assembled in house.

Before each experiment, we localized the focal point in the MRI coordinate space by visualizing heating in a silicone phantom (Reston, $3 \mathrm{M}$ ) using temperature-sensitive MRI. The anesthetized rat was then placed on the system and standard anatomical MR images were obtained to choose the targets. Sonications were applied in a $2 \times 2$ grid (spacing: $1 \mathrm{~mm}$ ) centered on either the right thalamus or the right putamen. Each sonication was preceded by a bolus injection of Optison (dose: $100 \mu \mathrm{l} / \mathrm{kg}$ ). We waited 1-2 minutes between sonications to allow the bubbles to clear from circulation. After each sonication, a 3D T2*weighted FLASH sequence (TR/TE 43/16 msec, flip angle $15^{\circ}$, field of view [FOV], $4 \times 4 \times 1.3 \mathrm{~cm}$, matrix $128 \times 128$ $\times 13$, slice thickness $1.0 \mathrm{~mm}$, average 1 ) was used to detect extravasated red blood cells produced by the sonications. After the 4 targets were sonicated, we obtained T2weighted fast spin echo (FSE) images (TR/TE 2000/68 msec, echo train length [ETL] 8, FOV $4 \mathrm{~cm}$, matrix $128 \times$ 128, slice thickness $1.5 \mathrm{~mm}$, averages 2). T1-weighted FSE images (TR/TE 500/18.6 msec, ETL 4, FOV $4 \mathrm{~cm}$, matrix $128 \times 128$, slice thickness $1.5 \mathrm{~mm}$, averages 4) were acquired before and after an intravenous injection of the MRI contrast agent Gd-DTPA (Magnevist, Berlex).

In 13 rats, the lesion development over time was monitored in MRI. Postsonication imaging in these sessions consisted of T1-, T2- and T2*-weighted images obtained without MRI contrast. This imaging was performed at Week 2 or 3 and again at Week 4 or 5 in all animals. In 7 animals, additional imaging was performed at Week 6 or 7, and in 1 animal, at Week 9 as well. Two animals were sacrificed at 4 days to examine short-term tissue effects.

\section{Histological Examination}

At 1-2 hours after the last MRI session, the animals were deeply anesthetized, and they were then killed and their brains were fixed via transcardial perfusion $(0.9 \%$ $\mathrm{NaCl}, 250 \mathrm{ml} ; 10 \%$ buffered formalin phosphate, $500 \mathrm{ml}$ ). The brains were removed and placed in $10 \%$ buffered formalin phosphate for immersion fixation. The brains were cut into 2- to 4-mm blocks with a rat brain matrix (model RBM-4000DV, ASI Instruments). Macrophotographs were obtained of both sides of each block. Representative examples $(n=8)$ were then embedded in paraffin and serially sectioned at $5 \mu \mathrm{m}$. Slides were stained with hematoxylin and eosin (H \& E) and Luxol fast blue (LFB) for light microscopy evaluation of the resulting lesions. The sections were scanned at $20 \times$ or $40 \times$ using a microscope slide scanner.

\section{Data Analysis}

The images were manually segmented by 1 investigator (N.M.) to quantify the size of the ablated regions at the different time points. Since in most cases the lesions appeared in MRI to be merged with the ventricles, it was not possible to clearly delineate their margins in many of the images. Thus, only a single image oriented perpendicular to the direction of the ultrasound beam was considered. This image corresponded to the focal plane, or an image $\pm 1 \mathrm{~mm}$ away. The area of the ablated regions were compared with each other using a paired Student t-test, with $\mathrm{p}$ $<0.05$ considered significant.

\section{Results}

MRI

The lesions were evident immediately after each sonication in $\mathrm{T} 2 *$-weighted images as hypointense spots (Fig. 2). As the 4 overlapping targets were sonicated, these hypointense spots merged into a single volume. Figures 3 and 4 show 2 examples of the lesion progression over time in different MRI sequences. Shortly after sonication, the sonicated volume appeared as a heterogeneous but slightly hypointense area in T2-weighted images surrounded by a

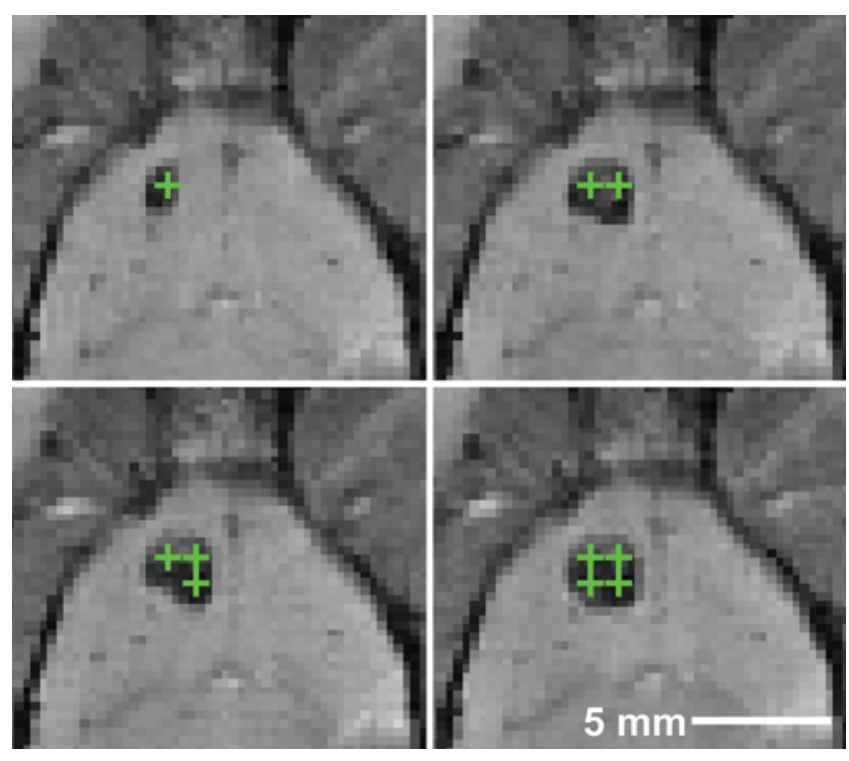

FIG. 2. T2*-weighted MR images showing hypointense spots produced immediately after each sonication. Four overlapping targets (spacing: $1 \mathrm{~mm}$ ) were sonicated to ablate a volume in the putamen. The ablated area partially overlapped the genu of the corpus callosum. Figure is available in color online only. 

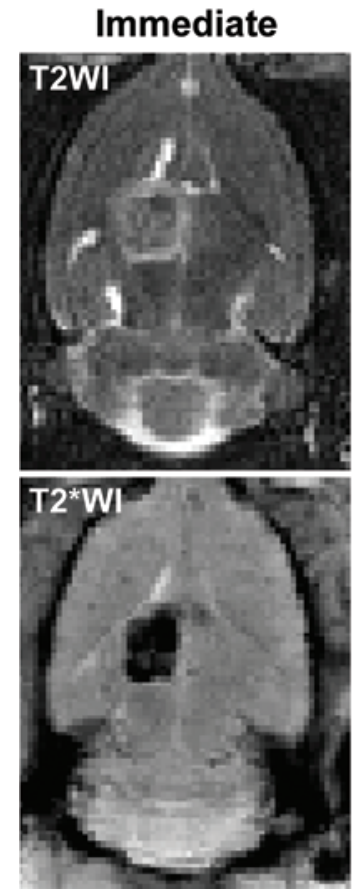

2 weeks
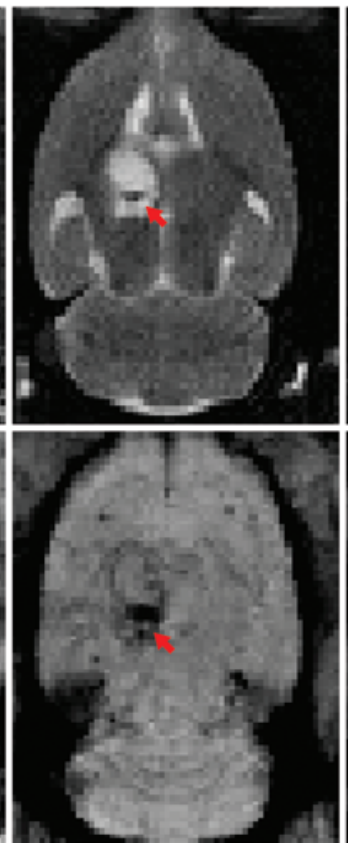

4 weeks
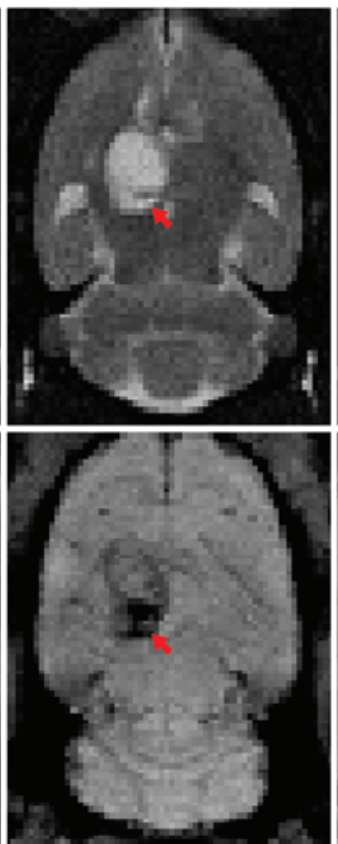

7 weeks
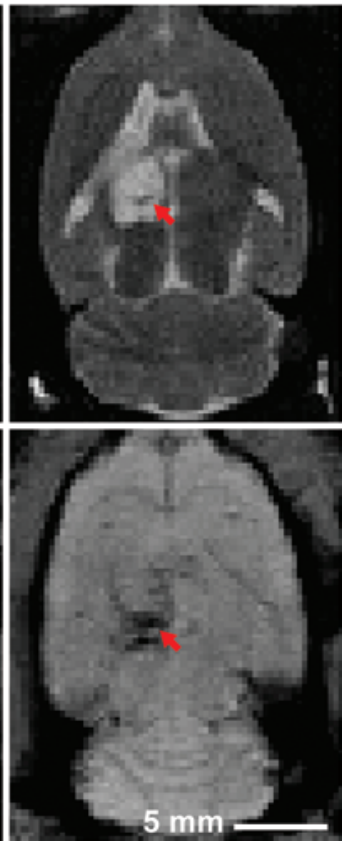

FIG. 3. Axial MR images acquired immediately after FUS ablation and 2, 4, and 7 weeks later. Immediately after sonication, the ablated region appeared as a slightly hypointense area surrounded by a hyperintense rim in T2-weighted MRI (T2WI). Two weeks later, the sonicated volume was mostly uniform and hyperintense, presumably indicating the production of a fluid-filled cyst. The size and appearance did not vary significantly at Week 4 or 7, although some enlargement of the lateral ventricle was observed. In T2*-weighted MRI (T2*WI), the ablated region was initially hypointense. At Weeks 2-4, the outer rim of the lesion was slightly hypointense. A residual dark spot (arrows) was present in both T2- and T2*-weighted imaging. Figure is available in color online only.

hyperintense rim. At Week 3 and thereafter, the lesion appeared uniform and hyperintense, presumably indicating the production of a sharply defined cyst-like region filled with CSF. In some animals, distinctly hypointense regions were evident at the lesion margin (arrows in Fig. 3). Over the next 2-9 weeks, the size of the lesion was stable or decreased by a small amount. The lateral ventricles were enlarged in most cases, but in no case did the lesion appear to grow over time.

In most of the rats, the lesion appearance in the T2*weighted images was similar to that shown in Figs. 3 and 4: the lesion center had a similar intensity as the CSF, and the outer rim of the lesion was hypointense. In some animals, however, areas within the lesion remained hypointense until the last imaging session. The lesions showed enhancement in T1-weighted images obtained after injection of Gd-DTPA MRI contrast agent, with the greatest enhancement at the margins (Fig. 4). At later times, the lesion was uniformly hypointense.

The length of the lesions along the direction of the ultrasound beam traversed the entire thickness of the brain. However, large white matter tracts that were included in the sonicated volume often remained intact in MR images for the duration of the experiments, even when the surrounding tissue was completely removed. In several cases, the intact corpus callosum or optic tract traversed the cystlike region and appeared to be floating in the CSF. An example of this is indicated by the arrows in Fig. 4.

The cross-sectional area of the ablated regions in the focal plane was measured by manually segmenting the hyperintense regions evident in T2-weighted images and the hypointense regions in the $\mathrm{T} 2 *$-weighted images. The results of this analysis are summarized in Fig. 5. Immediately after the sonications, the hyperintense regions in the T2-weighted images were larger $(\mathrm{p}<0.01)$ than the hypointense region in the $\mathrm{T} 2 *$-weighted images. Two weeks later, the lesion area in T2-weighted images was reduced substantially and appeared to be stable over the following 2-7 weeks (Fig. 5 lower). In many animals the hypointense area in $\mathrm{T} 2 *$-weighted images obtained immediately after sonication appeared to be predictive of the area of the cyst-like regions seen in the T2-weighted images obtained at Weeks $2-9$. The difference between these 2 measurements was not significant $(\mathrm{p}=0.12)$. However, in several cases (note Rats 1, 7, 9, and 12 in Fig. 5 upper), the size of the cyst in the T2-weighted images at 2 weeks and onward was markedly less than the initial size in the $\mathrm{T} 2 *$-weighted images.

\section{Histological Examination}

In the 2 animals sacrificed 4 days after microbubbleenhanced FUS ablation, a well-defined area with a sharp boundary was present at the targeted regions. The ablated areas exhibited a liquefaction phase of a cerebral necrosis (infarction), as shown in Fig. 6. At this stage the infarcted area was occupied by a homogeneous mass of necrotic tissue where almost no residual tissue architecture was preserved. Surrounding this region was a partly cystic 
Immediately post-FUS
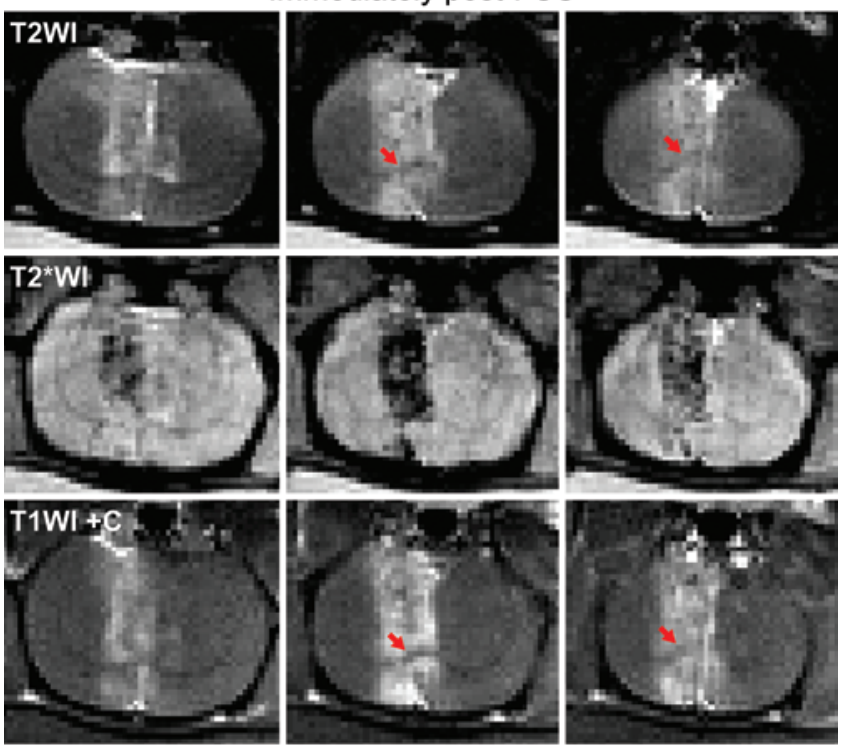

Six weeks post-FUS
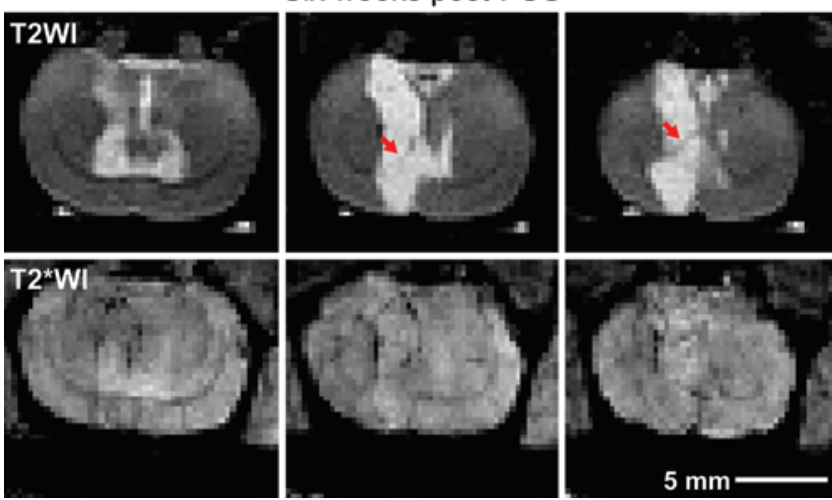

FIG. 4. Coronal MR images acquired immediately after sonication and 6 weeks later. Each row shows 3 neighboring image planes with the middle image centered on the ablated area. Immediately after sonication, the lesion was clearly evident as a hyperintense region in T2-weighted imaging and as a starkly hypointense area in $\mathrm{T}^{*}$-weighted imaging. On T1-weighted images obtained after administration of a contrast agent $(\mathrm{T} 1 \mathrm{WI}+\mathrm{C})$, signal enhancement was evident throughout the lesion except at the corpus callosum (arrows). Six weeks later, all that remained was a fluid-filled region in T2-weighted imaging and a narrow hypointense rim in T2*-weighted imaging. The corpus callosum appeared partially intact. Figure is available in color online only.

zone infiltrated by numerous macrophages phagocytizing degenerate material (Fig. 6C). The outer edge of the lesion was sharply defined (Fig. 6D). The size of the lesion observed in histological examination was similar to that seen in T2-weighted MR images shortly after sonication (inset in Fig. 6B).

At 4-9 weeks, the ablated areas appeared in the formalin-fixed blocks as empty cavities. In some cases the lining of these cavities was brownish-orange in color, but in most cases the lining was the same color as the brain tissue. White matter tracts were often intact and bridged these open cavities. An example of this phenomenon is shown in Fig. 7, where a white matter tract spans the empty cavity produced by the sonications.
Photomicrographs of the $\mathrm{H} \& \mathrm{E}-\mathrm{LFB}$-stained brain sections obtained at 4-9 weeks after FUS revealed cystic infarcts containing a central cavity surrounded by the infiltrating macrophages and astroglial cells. Figure 8 shows an infarction in the hippocampus (more dorsal slice from the same animal shown in Fig. 7) resulting in a large cystlike cavity lined by a narrow rim of macrophages containing hemosiderin and individual mineralization spots (Fig. 8D). However, the white matter band (accumulation of myelinated axons along the edge of the hippocampus) appeared mostly intact (Fig. 8E).

A second example from an animal sacrificed 6 weeks after sonication is shown in Fig. 9. Coronal views in MRI, gross pathological examination, and H \& E-LFB photomicrographs showed a cystic cavity traversing almost the entire thickness of the brain (Fig. 9A-C, respectively). Unlike in the previous example, the portion of the corpus callosum that was included in the sonicated area in this animal was destroyed (Fig. 9D), with a sharply-defined boundary. A thin membrane containing macrophages was observed on the brain surface and enclosed the cystic region (Fig. 9E).

\section{Discussion}

In this study, we sonicated overlapping targets to ablate a larger volume that covered the entire thickness of the brain from the skull base to the cortex. The ablated volume was large enough to result in a stable, cyst-like cavity that often included the ventricle and large white matter tracts. The present work builds upon our previous study in rats, where we sonicated a single brain target that overlapped or was adjacent to the optic chiasm and showed using visually evoked potential measurements that visual function was not significantly affected ${ }^{30}$ In that study, a comparatively small lesion was made, and in most cases only a scar was evident in histology at one month. We did not examine the lesion development over time, and we did not examine the cumulative effects of sonicating multiple targets. Here, serial MRI was performed to examine the lesion development over time to ensure that delayed effects did not occur. Overall, the results of the present work continue to support further advancement of this ablation approach to enable the use of transcranial FUS throughout the brain.

Tissue necrosis after ischemic stroke occurs over stages, ranging from a few minutes to days or weeks after the initial infarct. ${ }^{11,17}$ With nonthermal FUS ablation, the interaction between the circulating microbubbles and the ultrasound field produces mechanical stresses on the microvasculature that result in the halting of the blood supply in the capillary bed, leading to a localized infarct and tissue death. Presumably, the progression of the tissue necrosis in the sonicated region will follow a similar course as ischemic stroke. Our results are consistent with this timeline.

We did not observe delayed effects that might be expected to be a risk with this type of ablation. For example, we did not observe delayed hemorrhage that might be produced if a large blood vessel was damaged by the sonication and failed at a later time. We also did not observe 

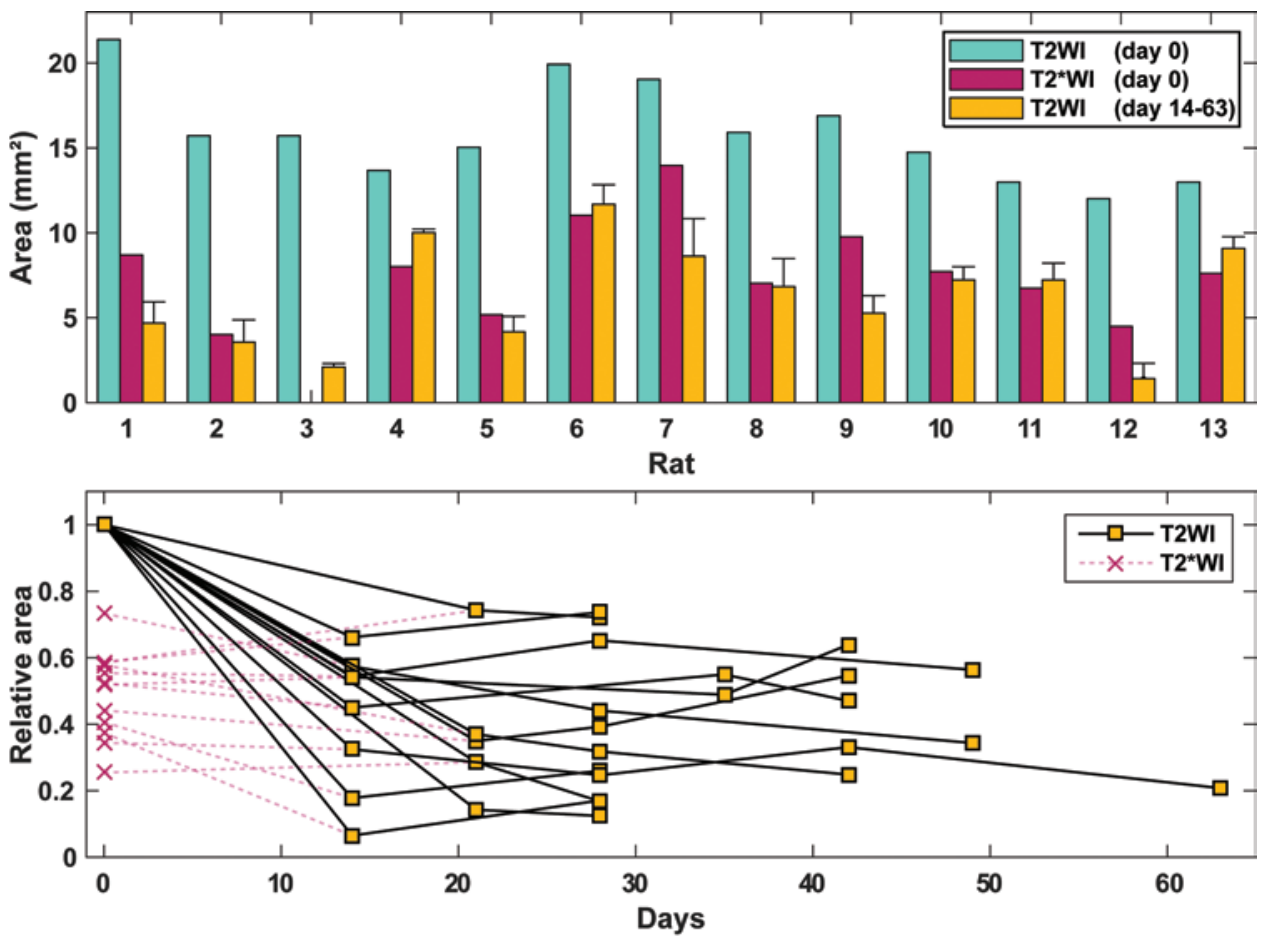

FIG. 5. Cross-sectional area of the lesions measured in the focal plane in MRI shortly after sonication (Day 0) and at later times. The areas were obtained by manually segmenting the hyperintense regions evident in T2-weighted imaging and the hypointense regions evident in $\mathrm{T} 2^{*}$-weighted imaging for each animal. Areas in T2*-weighted images were measured only at Day 0 . Upper: Graph showing these areas for each rat, with average areas (mean \pm SD) shown for the T2-weighted images at 14 days and onward. The areas in T2-weighted images were significantly reduced after Day 0 . In most cases the areas in T2*-weighted images were similar to the final lesion size in T2-weighted images. However, in 4 animals (Rats 1, 7, 9, and 12), the final areas in the T2-weighted images were markedly smaller. Lower: Plot of the area measured at each imaging session as a function of time for each rat. The areas are shown relative to their initial value in the T2-weighted images as a function of time after sonication. The relative area in T2*-weighted imaging is shown for reference. In each animal, the affected area in the T2-weighted images was largely reduced by Day 14 and was mostly stable thereafter. In no case was significant lesion growth observed. Figure is available in color online only.
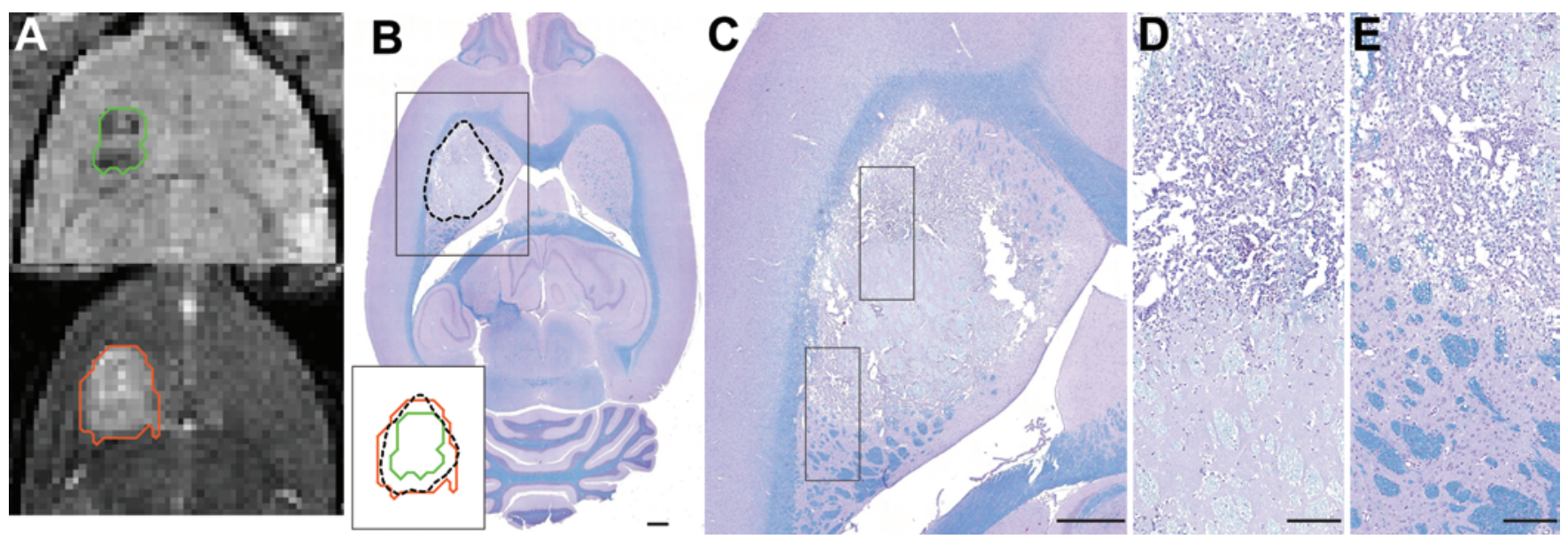

FIG. 6. MR images and photomicrographs of an $\mathrm{H}$ \& E-LFB-stained section obtained after microbubble-enhanced FUS ablation in the putamen. A: Axial T2*-weighted (upper) and T2-weighted (lower) MR images acquired immediately after sonication. The ablated area was segmented in each image. B-E: Photomicrographs of an H \& E-LFB stained section obtained 4 days after sonication. A well-defined area was present at the targeted region $(B-C)$. Segmentations from the different images revealed that the size of the lesion in histology closely matched the hyperintense region in T2-weighted imaging (inset). Macrophage infiltration was evident at the edge of the lesion (D). This infiltration had not reached the center core of the lesion, where both gray matter and white matter structures appeared pale-stained and acellular. A sharp boundary was found between the outer edge of the lesion and the normal brain (E). Bar $=1 \mathrm{~mm}(B$ and $C), 200 \mu \mathrm{m}$ (D and E). Figure is available in color online only. 

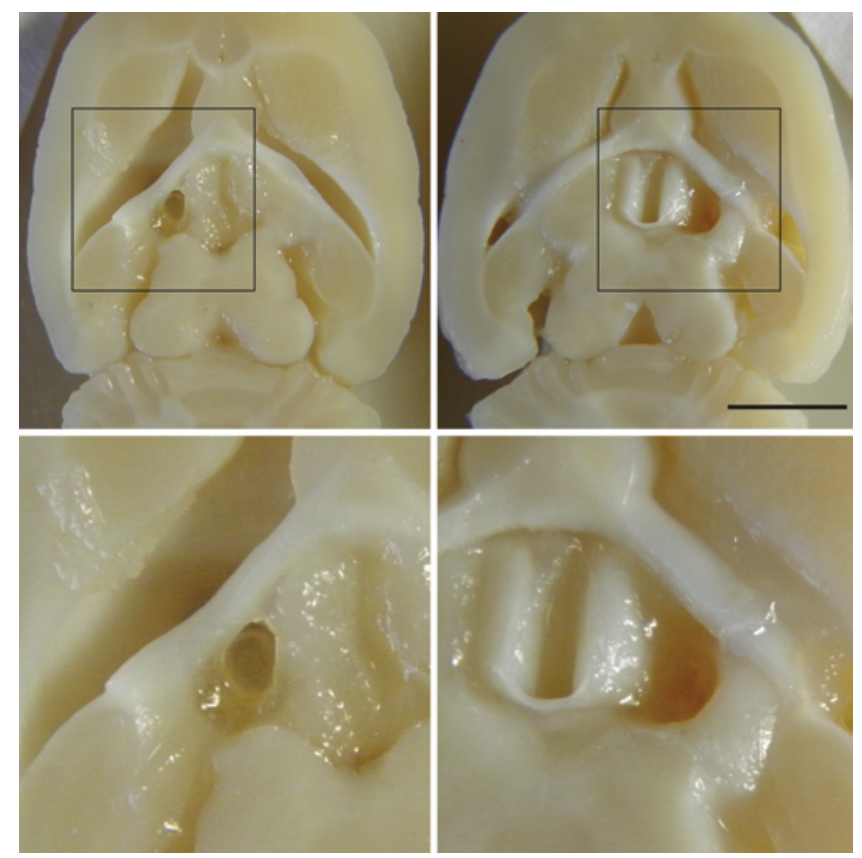

FIG. 7. Photograph showing 2 adjacent formalin-fixed blocks of a rat brain obtained 9 weeks after FUS ablation. An empty cavity was observed at the ablation site in the thalamus. A large white matter tract that was included in the sonicated region appeared intact. Bar $=5 \mathrm{~mm}$. Figure is available in color online only.

enlargement of the lesion over time, which might occur when the necrotic tissue was broken down and removed. From 2 weeks onward the lesions appeared stable in MR images as well-defined areas filled with CSF. In each animal, the infarcted area was large and had glial proliferation (gliosis), a regular process that is intended to fill in the infarcted territory and form a glial scar. Here, it was not strong enough to fill the defect; a cyst-like cavity remained lined by astrocytic tissue.

In some cases, large white matter structures that were included in the sonicated volume appeared to remain partially intact, even when all of the surrounding tissue was removed. These structures often were observed to be floating like a bridge across the cavity where the ablated tissue was removed. This finding is consistent with prior work with this method ${ }^{30}$ and likely stems from the relative paucity of blood vessels in white matter compared with the highly vascular gray matter. In some cases, however, large white matter structures were ablated, with sharply defined boundaries. Such cases suggest that multiple sonications could be required to ablate large white matter tracts. However, more work is needed to understand what circumstances lead to destruction of white matter tracts.

In the 2 animals killed four days after sonication, the size of the lesion in histological sections was similar to the hyperintense region in T2-weighted imaging acquired immediately after sonication. This hyperintense area was larger than the hypointense region observed in $\mathrm{T} 2 *$-weighted images. While more tests are needed, these results perhaps imply that the hyperintense area in T2-weighted MRI (edematous, presumably) represents the extent of the necrosis and that this area was larger than the areas where

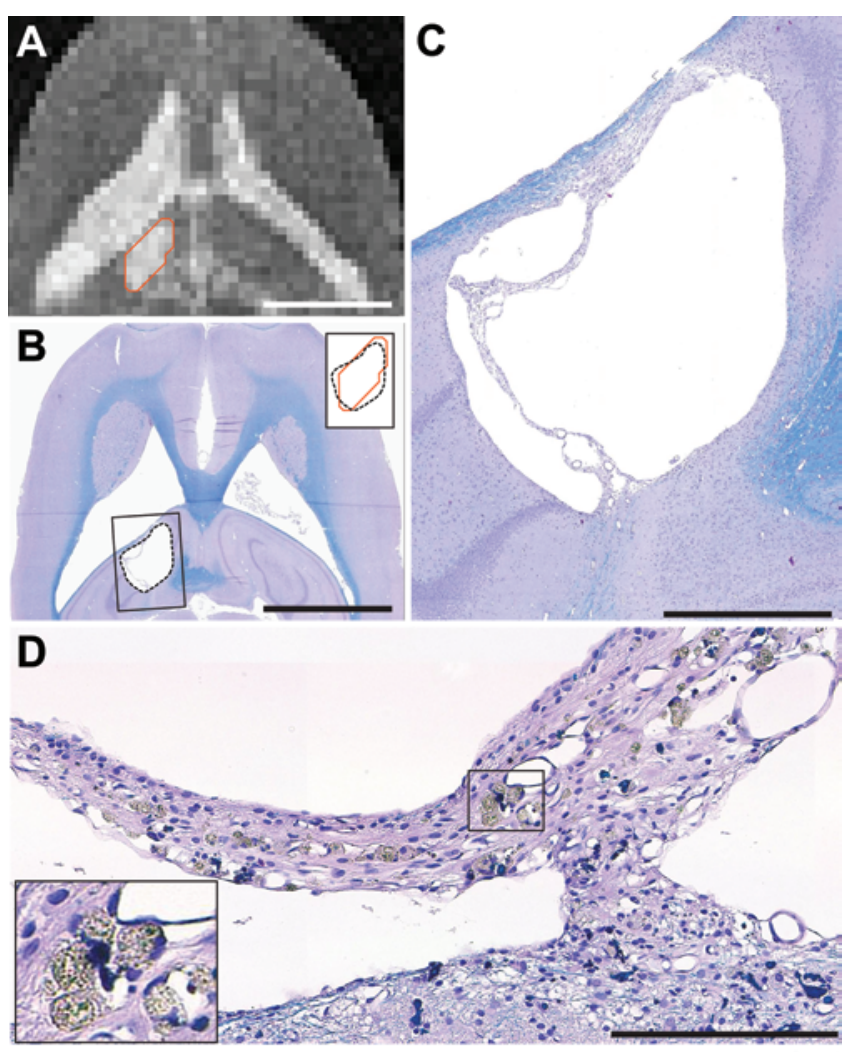

E

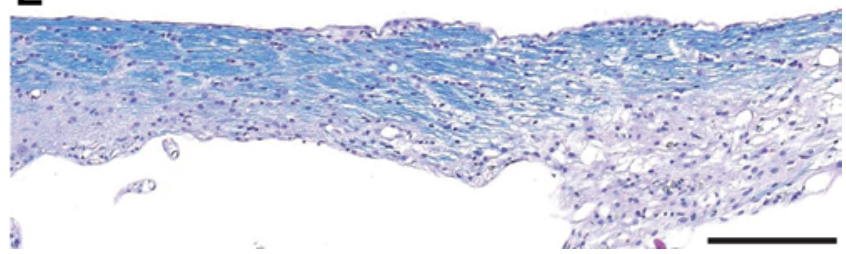

FIG. 8. MR images and photomicrographs of $H$ \& E-LFB-stained sections of the brain shown in Fig. 7 obtained 9 weeks after FUS ablation. A-C: A cyst-like region was observed at the sonication site in both $\mathrm{MRI}$ and histological examination along with enlarged ventricles. Superimposed segmentations (inset in B) show some distortion, but similar areas evident in MRI and stained section. D: A higher-magnification view of the lesion edge from an adjacent section shows a sharp boundary with a narrow rim of infiltrating macrophages containing hemosiderin (brown) and mineralization spots (dark blue). E: A white matter tract included in the sonicated region appears damaged but partially intact. Bar $=5 \mathrm{~mm}(A$ and $B), 1 \mathrm{~mm}(C)$, and $200 \mu \mathrm{m}(D-E)$. Figure is available in color online only.

the mechanical stresses on the microvasculature produced microhemorrhages evident as hypointense regions in $\mathrm{T} 2 *_{-}$ weighted images. We cannot say whether the edematous zone is a direct effect of the sonication or a side-effect of the adjacent damaged/hemorrhagic vasculature. It would be interesting to investigate whether lower exposure levels that do not produce these microhemorrhages can directly induce tissue necrosis.

In most of the animals, the area of the cystic lesion in T2-weighted imaging at 2 weeks after sonication and later was close to that of the hypointense region in $\mathrm{T} 2 *_{\text {- }}$ weighted images acquired immediately after the sonications. This finding might suggest that the hemorrhagic 


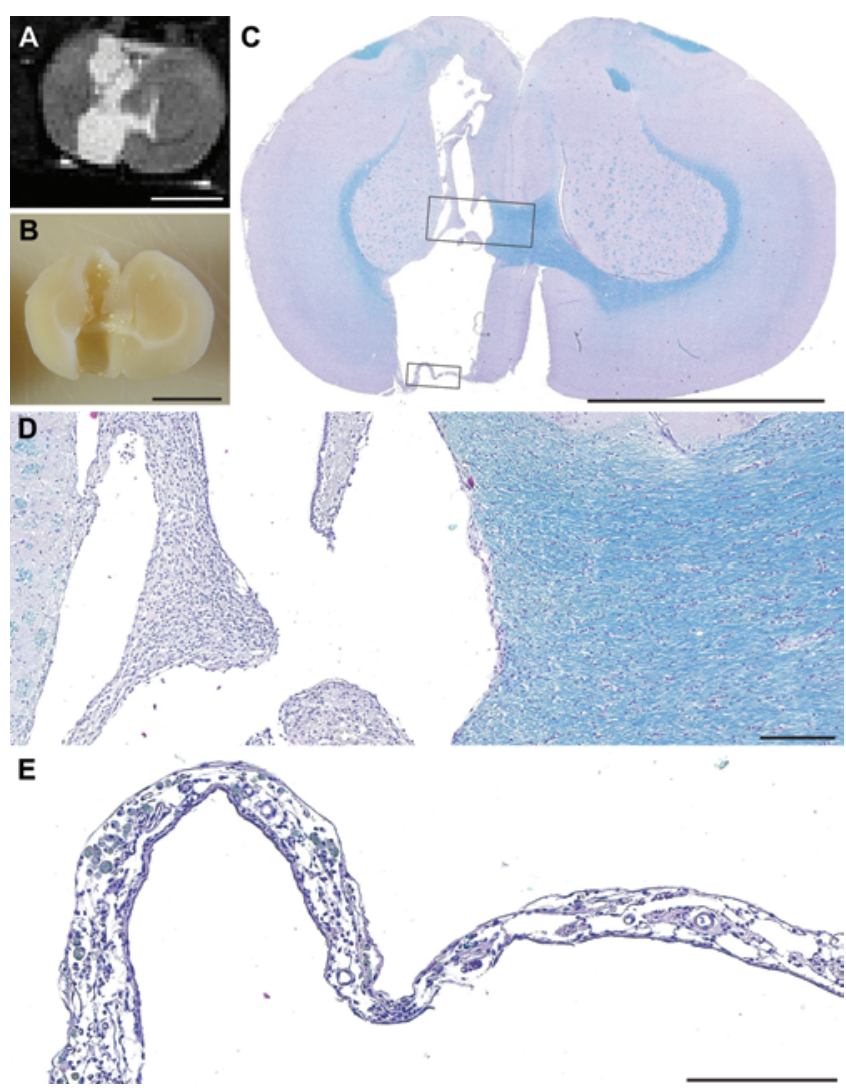

FIG. 9. Coronal MR image, photograph of formalin-fixed block, and photomicrographs of $\mathrm{H}$ \& E-LFB-stained sections obtained 6 weeks after microbubble-enhanced FUS ablation. A-C: A cyst-like volume was evident in T2-weighted MRI, in the tissue block, and in $\mathrm{H}$ \& E-LFB stained sections. The volume included a portion of the corpus callosum, which was severed in this example. D: The boundary of the severed corpus callosum was sharply defined. E: The FUS beam propagated from below, and the lesion extended to the surface of the brain. A thin membrane containing macrophages enclosed the cystic region. Bar $=5$ $\mathrm{mm}(\mathrm{A}-\mathrm{C})$ and $200 \mu \mathrm{m}(\mathrm{D}-\mathrm{E})$. Figure is available in color online only.

area becomes cystic and the necrotic edematous area is resorbed. It is also possible that this agreement was coincidental and simply reflects tissue shrinkage as some of the necrotic tissue is resorbed and becomes cystic. More studies are needed where histological specimens are acquired at earlier times to understand the progression of the different zones within the lesions and how they relate to MRI findings.

The transducer used in this study had a long focal area that covered the entire thickness of the rat brain in the direction of the ultrasound beam propagation. As a consequence, we could not examine whether such lesions could be produced without damaging tissues along the ultrasound beam path. It is possible that the exposure levels used here would not be possible at deep targets in a human without exceeding the threshold for inertial cavitation in the beam path and that lower acoustic pressure amplitudes would need to be employed. In a recent study in nonhuman primates, disruption of the blood-brain barrier and minor tissue damage occurred in the ultrasound beam path, suggesting a need for careful control of the exposure level.
Additional work is required to ensure that unexpected results do not occur when the exposure level is lower.

The relatively low frequency used here along with the long focal area also likely resulted in strong reflections from the skull base and the formation of standing waves within the intact rat skull. ${ }^{34}$ These effects, along with differences in skull thickness, likely caused uncertainty in the pressure amplitude in the brain and the variability in lesion size that was observed in Fig. 5. Other sources of error included uncertainty in the lesion measurements due to their overlapping with the ventricles as well as relatively low-resolution MR imaging, which may have prevented us in detecting small changes in lesion size over time. Finally, the issue of how to monitor and control the sonications was not examined here. While MRI was able to show the lesions immediately after the each sonication (Fig. 2), real-time methods are needed. We anticipate that passive acoustic mapping methods ${ }^{16,37,39}$ can be useful for this purpose for transcranial FUS. ${ }^{1,35}$

\section{Conclusions}

These findings suggest that nonthermal ablation achieved using low-intensity FUS bursts and a circulating microbubble ultrasound contrast agent can produce cystic lesions within 2 weeks after sonication that are stable over time. We did not find delayed hemorrhages or other unexpected effects, and the lesions did not increase in size over time. These results are encouraging for the prospect of this technique to expand the "treatment envelope" of transcranial FUS ablation, as 4 overlapping sonications were used to produce volumetric lesions of significant volume with respect to the size of the rat brain. Future work should explore whether lower exposure levels can be used to achieve nonthermal ablation and to better understand the effects in white matter tracts, which appeared relatively immune to the sonication effects in many, but not all, cases.

\section{Acknowledgments}

This work was supported by NIH grants P01CA174645 and P41EB015898.

\section{References}

1. Arvanitis CD, McDannold N: Integrated ultrasound and magnetic resonance imaging for simultaneous temperature and cavitation monitoring during focused ultrasound therapies. Med Phys 40:112901, 2013

2. Aubry JF, Tanter M, Pernot M, Thomas JL, Fink M: Experimental demonstration of noninvasive transskull adaptive focusing based on prior computed tomography scans. J Acoust Soc Am 113:84-93, 2003

3. Ballantine HT Jr, Bell E, Manlapaz J: Progress and problems in the neurological applications of focused ultrasound. $\mathbf{J}$ Neurosurg 17:858-876, 1960

4. Bauer R, Martin E, Haegele-Link S, Kaegi G, von Specht $\mathrm{M}$, Werner B: Noninvasive functional neurosurgery using transcranial MR imaging-guided focused ultrasound. Parkinsonism Relat Disord 20 (Suppl 1):S197-S199, 2014

5. Burke CW, Klibanov AL, Sheehan JP, Price RJ: Inhibition of glioma growth by microbubble activation in a subcutaneous model using low duty cycle ultrasound without significant heating. J Neurosurg 114:1654-1661, 2011

6. Chung DJ, Cho SH, Lee JM, Hahn ST: Effect of microbubble 
contrast agent during high intensity focused ultrasound ablation on rabbit liver in vivo. Eur J Radiol 81:e519-e523, 2012

7. Clement GT, Hynynen K: A non-invasive method for focusing ultrasound through the human skull. Phys Med Biol 47:1219-1236, 2002

8. Clement GT, Sun J, Giesecke T, Hynynen K: A hemisphere array for non-invasive ultrasound brain therapy and surgery. Phys Med Biol 45:3707-3719, 2000

9. Coluccia D, Fandino J, Schwyzer L, O'Gorman R, Remonda L, Anon J, et al: First noninvasive thermal ablation of a brain tumor with MR-guided focused ultrasound. J Ther Ultrasound 2:17, 2014

10. Elias WJ, Huss D, Voss T, Loomba J, Khaled M, Zadicario E, et al: A pilot study of focused ultrasound thalamotomy for essential tremor. N Engl J Med 369:640-648, 2013

11. Fisher M, Bastan B: Identifying and utilizing the ischemic penumbra. Neurology 79 (13 Suppl 1):S79-S85, 2012

12. Flynn HG, Church CC: A mechanism for the generation of cavitation maxima by pulsed ultrasound. J Acoust Soc Am 76:505-512, 1984

13. Fujishiro S, Mitsumori M, Nishimura Y, Okuno Y, Nagata Y, Hiraoka M, et al: Increased heating efficiency of hyperthermia using an ultrasound contrast agent: a phantom study. Int J Hyperthermia 14:495-502, 1998

14. Goertz DE, Todorova M, Mortazavi O, Agache V, Chen B, Karshafian R, et al: Antitumor effects of combining docetaxel (taxotere) with the antivascular action of ultrasound stimulated microbubbles. PLoS One 7:e52307, 2012

15. Goss SA, Frizzell LA, Dunn F: Ultrasonic absorption and attenuation in mammalian tissues. Ultrasound Med Biol 5:181-186, 1979

16. Gyöngy M, Coussios CC: Passive cavitation mapping for localization and tracking of bubble dynamics. J Acoust Soc Am 128:EL175-EL180, 2010

17. Heiss WD: The ischemic penumbra: how does tissue injury evolve? Ann N Y Acad Sci 1268:26-34, 2012

18. Huang Y, Hynynen K: MR-guided focused ultrasound for brain ablation and blood-brain barrier disruption. Methods Mol Biol 711:579-593, 2011

19. Huang Y, Vykhodtseva NI, Hynynen K: Creating brain lesions with low-intensity focused ultrasound with microbubbles: a rat study at half a megahertz. Ultrasound Med Biol 39:1420-1428, 2013

20. Hynynen K: Biophysics and technology of ultrasound hyperthermia, in Gautherie M (ed): Methods of External Hyperthermic Heating. Berlin: Springer, 1990, pp 61-115

21. Ishihara Y, Calderon A, Watanabe H, Okamoto K, Suzuki Y, Kuroda K, et al: A precise and fast temperature mapping using water proton chemical shift. Magn Reson Med 34:814823,1995

22. Jeanmonod D, Werner B, Morel A, Michels L, Zadicario E, Schiff G, et al: Transcranial magnetic resonance imagingguided focused ultrasound: noninvasive central lateral thalamotomy for chronic neuropathic pain. Neurosurg Focus 32(1):E1, 2012

23. Jolesz FA, McDannold NJ: Magnetic resonance-guided focused ultrasound: a new technology for clinical neurosciences. Neurol Clin 32:253-269, 2014

24. Jung HH, Chang WS, Rachmilevitch I, Tlusty T, Zadicario E, Chang JW: Different magnetic resonance imaging patterns after transcranial magnetic resonance-guided focused ultrasound of the ventral intermediate nucleus of the thalamus and anterior limb of the internal capsule in patients with essential tremor or obsessive-compulsive disorder. J Neurosurg 122:162-168, 2015

25. Kim YZ, Kim DY, Yoo H, Yang HS, Shin SH, Hong EK, et al: Radiation-induced necrosis deteriorating neurological symptoms and mimicking progression of brain metastasis after stereotactic-guided radiotherapy. Cancer Res Treat 39:16-21, 2007

26. Lele PP: Effects of ultrasound on "solid" mammalian tissues and tumors in vivo, in Repacholi MH, Grondolfo M, Rindi A (eds): Ultrasound: Medical Applications, Biological Effects, and Hazard Potential. New York: Plenum, 1987, pp 275-306

27. Lipsman N, Schwartz ML, Huang Y, Lee L, Sankar T, Chapman M, et al: MR-guided focused ultrasound thalamotomy for essential tremor: a proof-of-concept study. Lancet Neurol 12:462-468, 2013

28. Martin E, Jeanmonod D, Morel A, Zadicario E, Werner B: High-intensity focused ultrasound for noninvasive functional neurosurgery. Ann Neurol 66:858-861, 2009

29. McDannold N, Clement GT, Black P, Jolesz F, Hynynen K: Transcranial magnetic resonance imaging- guided focused ultrasound surgery of brain tumors: initial findings in 3 patients. Neurosurgery 66:323-332, 2010

30. McDannold N, Zhang YZ, Power C, Jolesz F, Vykhodtseva $\mathrm{N}$ : Nonthermal ablation with microbubble-enhanced focused ultrasound close to the optic tract without affecting nerve function. J Neurosurg 119:1208-1220, 2013

31. McDannold NJ, Vykhodtseva NI, Hynynen K: Microbubble contrast agent with focused ultrasound to create brain lesions at low power levels: MR imaging and histologic study in rabbits. Radiology 241:95-106, 2006

32. Meyers R, Fry WJ, Fry FJ, Dreyer LL, Schultz DF, Noyes RF: Early experiences with ultrasonic irradiation of the pallidofugal and nigral complexes in hyperkinetic and hypertonic disorders. J Neurosurg 16:32-54, 1959

33. Miller DL, Gies RA: Enhancement of ultrasonically-induced hemolysis by perfluorocarbon-based compared to air-based echo-contrast agents. Ultrasound Med Biol 24:285-292, 1998

34. O'Reilly MA, Huang Y, Hynynen K: The impact of standing wave effects on transcranial focused ultrasound disruption of the blood-brain barrier in a rat model. Phys Med Biol 55:5251-5267, 2010

35. O'Reilly MA, Jones RM, Hynynen K: Three-dimensional transcranial ultrasound imaging of microbubble clouds using a sparse hemispherical array. IEEE Trans Biomed Eng 61:1285-1294, 2014

36. O'Reilly MA, Muller A, Hynynen K: Ultrasound insertion loss of rat parietal bone appears to be proportional to animal mass at submegahertz frequencies. Ultrasound Med Biol 37:1930-1937, 2011

37. Salgaonkar VA, Datta S, Holland CK, Mast TD: Passive cavitation imaging with ultrasound arrays. J Acoust Soc Am 126:3071-3083, 2009

38. Sanghvi NT, Fry FJ, Foster RS, Bihrle R, Zaitsev A, Hennige C: High intensity focused ultrasound treatment of prostate tissue in the presence of an US contrast agent. J Ultrasound Med 14 Suppl:S17, 1995

39. Sato T, Uemura K, Sasaki K: Super-resolution acoustical passive imaging system using algebraic reconstruction. J Acoust Soc Am 67:1802-1808, 1980

40. Tran BC, Seo J, Hall TL, Fowlkes JB, Cain CA: Microbubble-enhanced cavitation for noninvasive ultrasound surgery. IEEE Trans Ultrason Ferroelectr Freq Control 50:12961304,2003

41. Umemura S, Kawabata K, Hashiba K: Enhancement of ultrasonic absorption by microbubbles for therapeutic application. Proc 2001 IEEE Ultrasonics Symp 2:1311-1314, 2001

42. Vykhodtseva N, McDannold N, Hynynen K: Induction of apoptosis in vivo in the rabbit brain with focused ultrasound and Optison. Ultrasound Med Biol 32:1923-1929, 2006

43. Wang TY, Xu Z, Hall TL, Fowlkes JB, Cain CA: An efficient treatment strategy for histotripsy by removing cavitation memory. Ultrasound Med Biol 38:753-766, 2012 
44. White PJ, Clement GT, Hynynen K: Longitudinal and shear mode ultrasound propagation in human skull bone. Ultrasound Med Biol 32:1085-1096, 2006

45. Yu T, Wang G, Hu K, Ma P, Bai J, Wang Z: A microbubble agent improves the therapeutic efficiency of high intensity focused ultrasound: a rabbit kidney study. Urol Res 32:14-19, 2004

\section{Disclosures}

The authors report no conflict of interest concerning the materials or methods used in this study or the findings specified in this paper.

\section{Author Contributions}

Conception and design: McDannold. Acquisition of data: McDannold, Zhang. Analysis and interpretation of data: all authors.
Drafting the article: McDannold. Critically revising the article: Vykhodtseva. Reviewed submitted version of manuscript: McDannold, Vykhodtseva. Approved the final version of the manuscript on behalf of all authors: McDannold. Statistical analysis: McDannold.

\section{Supplemental Information}

\section{Previous Presentations}

Portions of this work were presented in abstract form at the Focused Ultrasound Surgery Foundation Symposium and at the International Society for Therapeutic Ultrasound.

\section{Correspondence}

Nathan McDannold, Department of Radiology, Brigham and Women's Hospital, Harvard Medical School, 75 Francis St., Boston, MA 02115.email: njm@bwh.harvard.edu. 